\title{
Impact of the 7-bp deletion in HvGA200x2 gene on agronomic important traits in barley (Hordeum vulgare L.)
}

Serafima Teplyakova ${ }^{1,2}$, Marina Lebedeva ${ }^{2,3}$, Nadezhda Ivanova², Valentina Horeva², Nina Voytsutskaya², Olga Kovaleva ${ }^{2}$ and Elena Potokina ${ }^{1,2,3^{*}}$

From 4th International Scientific Conference "Plant Genetics, Genomics, Bioinformatics and Biotechnology" (PlantGen 2017) Almaty, Kazakhstan. 29 May - 2 June 2017

\begin{abstract}
Background: Alike to Reduced height-1 (Rht-1) genes in wheat and the semi dwarfing (sd-1) gene in rice, the sdw1/denso locus involved in the metabolism of the GA, was designated as the 'Green Revolution' gene in barley. The recent molecular characterization of the candidate gene HVGA200x2 for sdw1/denso locus allows to estimate the impact of the functional polymorphism of this gene on the variation of agronomically important traits in barley.

Results: We investigated the effect of the 7-bp deletion in exon 1 of HvGA20ox2 gene (sdw1.d mutation) on the variation of yield-related and malting quality traits in the population of DHLs derived from cross of medium tall barley Morex and semi-dwarf barley Barke. Segregation of plant height, flowering time, thousand grain weight, grain protein content and grain starch was evaluated in two diverse environments separated from one another by $15^{\circ}$ of latitude. The 7-bp deletion in HvGA200x2 gene reduced plant height by approximately $13 \mathrm{~cm}$ and delayed flowering time by 3-5 days in the barley segregating DHLs population independently on environmental cue. On other hand, the $s d w 1 . d$ mutation did not affect significantly either grain quality traits (protein and starch content) or thousand grain weight.

Conclusions: The beneficial effect of the $s d w 1 . d$ allele could be associated in barley with lodging resistance and extended period of vegetative growth allowing to accumulate additional biomass that supports higher yield in certain environments. However, no direct effect of the $s d w 1 . d$ mutation on thousand grain weight or grain quality traits in barley was detected.
\end{abstract}

Keywords: Semi-dwarf barley, sdw1/denso, Functional polymorphism, QTL, Heading date, Plant height, Grain protein content, Thousand grain weight

\section{Background}

The tremendous success of the "Green Revolution" of the XX century was achieved due to the introduction in agriculture the new cereal varieties distinguished by a shortened thick stem, resistant to lodging. These varieties made it possible to change agricultural technology,

\footnotetext{
*Correspondence: e.potokina@vir.nw.ru

'Saint Petersburg State University, Universitetskaya emb.7/9, St. Petersburg 199034, Russia

${ }^{2}$ N.I. Vavilov Institute of Plant Genetic Resources (VIR), Bolshaya Morskaya, 42-44, 190000 St. Petersburg, Russia

Full list of author information is available at the end of the article
}

to increase the dose of mineral fertilizers, and, as a result, dramatically improved the productivity of cereal crops. For wheat and rice the "Green Revolution" genes were involved in a gibberellic acid (GA) metabolism influencing plant height (dwarfing), lodging resistance, and, consequently, harvest index (e.g. [1]). In hexaploid wheat, dwarfing has been achieved mainly through the introduction of the mutant alleles of Reduced height-1 $(R h t-1)$ genes that encode DELLA proteins, which act to repress GA-responsive growth [2]. In rice, the record yields obtained in the 1960s throughout Asia were owing to semi-dwarfing $s d-1$ mutants carrying a deletion of 
$280 \mathrm{bp}$ within the coding region of Os20ox2 gene resulting in the nonfunctional 20-oxidase GA biosynthetic enzyme [3]. Thus, the genes involved in the metabolism of GA (semi dwarfing $s d-1$ gene in rice and $R h t$ gene in wheat) have played the crucial role in plant height reduction, which led to a significant increase in yields of the two major cereal crops. In barley, however, the impact of the semi-dwarfing genes on agronomical success of modern varieties still remains uncertain.

Two dwarfing genes, $s d w 1 / d e n s o$ and uzu1.a involved, respectively, in the metabolism of the GA and brassinosteroid hormones, were designated as "the Green Revolution" genes in barley [4]. Among them, the $s d w 1$ barley gene is an ortholog of the semi-dwarf $s d-1$ gene in rice; the corresponding candidate gene HvGA20ox 2 encoding the gibberellin 20-oxidase was recently reported $[5,6]$. Four mutant alleles of $s d w 1$ gene are known in barley. One of them sdw1.c (originally named denso) was found in a spontaneous mutant selected from the $c v$. Abed Denso in Denmark. Three other $s d w 1$ alleles have been obtained artificially with the use of mutagens: 1) $s d w 1 . a$ mutant (originally named $s d w 1$ ) were induced by X-rays in the Norwegian six-rowed barley $c v$. Jotun. 2) the $s d w 1 . d$ mutant was obtained using X-rays treatment of cultivar Valticky and released in 1965 as $c v$. Diamant. Today cultivars with the $s d w 1 . d$ mutation from Diamant are often described as «sdw1/ denso». 3) sdw1.e named as 'Risø no. 9265' was isolated in a M2 generation from the variety Bomi treated by neutrons [7].

A pronounced success was achieved in molecular characterization of the $s d w 1$ barley alleles [5-8]. First, $H \nu G A 20 o x 2$ sequences of 2413 bp were compared between medium tall barley variety AC Metcalfe and semi-dwarf variety Baudin possessing the semidwarfing gene $(s d w 1 . d)$; the $\mathrm{A} / \mathrm{G}$ substitution was identified in the intron 2 co-segregating with plant height in AC Metcalfe/ Baudin DH progeny [5]. Next, the 7-bp deletion in exon 1 resulting in coding frame shifts of $H \nu G A 20 o x 2$ gene was suggested as the functional polymorphism of the $s d w 1 . d$ allele from Diamant [8]. A complete deletion of $H v G A 20 o x 2$ gene was identified in semi-dwarf mutant Riso no. 9265 carrying $s d w 1 . e$ allele, originated from the variety Bomi [7]. Five different sequence variations were identified by comparing the $H \nu G A 20 o x 2$ gene sequence of the $s d w 1 . c$ mutant (Abed Denso) with the tall barley cultivars: they include a 1bp deletion and a 4-bp insertion in the $5^{\prime}$ untranslated region as well as two synonymous mutations in coding sequence of $H v G A 20 o x 2$ gene [8]. While plant height variation was significantly associated with the 7-bp deletion of $H v G A 200 \times 2$ gene ( $s d w 1 . d$ allele) in a biparental mapping population and a natural population of barley varieties, none of the exposed sequence variations the sdw1.c mutants could explain dwarf phenotype [8].

All the $s d w 1$ barley mutants are distinguished by shorter and stronger culms supporting spikes and preventing lodging. However, the mutations in HvGA20ox2 gene affect biosynthesis of the GA that control many processes in different plant tissues, thus, their pleiotropic effects also cause a number of unwanted agronomic traits such as reduction of grain size [4]. Both beneficial and deleterious effects associated with $s d w 1$ gene were reported for barley (reviewed in [9]). Recent success in molecular characterization of the $s d w 1$ alleles facilitated further studies focusing on the better understanding of their pleiotropic effect on agronomic performance of barley varieties.

In the present paper we estimated the effect causing by the 7-bp deletion in exon 1 of $H v G A 20 o x 2$ gene (the $s d w 1 /$ denso allele) on the variation of economically important traits in the segregating population of the double haploid lines (DHLs) derived from the cross of the tall barley Morex and the semi-dwarf $s d w 1 . d$ mutant cv. Barke. Barke inherited the $s d w 1 /$ denso allele from Triumph via cv. Alexis (https://triticeaetoolbox.org/barley/), while Triumph is a descendant of cv. Diamant. We estimated the phenotypic effect of the $s d w 1 /$ denso allele in two very diverse environments by the QTL mapping approach. Variation of plant height, heading date, thousand grain weight, grain protein and starch content in seeds was evaluated.

\section{Methods}

Plant material and field evaluation

Seeds of double haploid lines (DHLs) derived from the cross between Morex and Barke were kindly offered for the field evaluation by Nils Stein (Gatersleben, Germany). The DHLs population is abbreviated further in the text as Morex/Barke DHLs population.

Field trials of the Morex/Barke DHLs population were conducted in two experimental stations of VIR: in Pushkin (59 $53^{\prime} 39$ "N, St. Petersburg region, North of Russia) and Krasnodar (45 $02^{\prime} 55$ "N, South of Russia). The field experiment consisted of two replicate plots each containing a row of 50 plants of each doubled haploid line and two rows of each parent. Rows were spaced approximately $20 \mathrm{~cm}$ apart.

DHLs were evaluated for heading date (HD) that was calculated as number of days from sowing until first awns visible. Plant height $(\mathrm{PH})$ was measured at full maturity: height to top of spike excluding awns was recorded for ten individuals per each $\mathrm{DH}$ line. $\mathrm{PH}$ records for Morex/Barke DHLs were obtained in 2015 (Pushkin).

Thousand grain weight (TGW) was measured at the conditioning humidity, according to standard protocols 
[10]: from the sample of grains for each $\mathrm{DH}$ line two weights were singled out, each with 500 seeds. The samples were weighed on the laboratory balances with an accuracy of $0.01 \mathrm{~g}$. The sum of the results of weighing of two samples of 500 seeds was calculated.

Grain Protein Content (GPC) and starch content in dry seeds were analyzed using Infratec 1241 Grain Analyser (Foss, USA). The data to construct the calibration curve for GPC were obtained with Kjeltec Auto 1030 Analyzer (Sweden) by Kjeldahl method. The calibration curve for starch determination was developed with Evers' Polarimetric Method.

\section{QTL mapping and statistical analysis}

For the Morex/Barke segregating population a comprehensive genetic map was available consisting of 1068 SNP markers for 93 DHLs [11], the published genotyping data were used for the QTL analysis. Mapping of QTL was carried out with the Windows QTL Cartographer version 2.5 software [12] using the CIM algorithm (Composite Interval Mapping). The minimum threshold of LOD, significant at 95\% ( $p=0.05)$, was calculated based on the results of 1000 permutations. To visualize LOD scans a Perl script was used (available upon request).

\section{CAPS assay to reveal 7 bp deletion in exon 1 of HvGA20ox2 gene}

PCR was performed with designed primers: forward $5^{\prime}$ CTCCCTCCCTCCCCGATTAC and reverse 5'-CCGG ACACCTGGAAGAACCC. The reaction mix contained $1 \times$ Taq-buffer (2.5 mM Mg2+); $200 \mu \mathrm{M}$ dNTP; 2,5 U Tag DNA polymerase (Sileks, Moscow); $0.4 \mu \mathrm{M}$ of each primer; 30 ng of template DNA, 5\% DMSO and sterile distilled water in a final volume of $25 \mu \mathrm{l}$. PCR cycling conditions consisted of an initial denaturation step of $95{ }^{\circ} \mathrm{C}$ for $3 \mathrm{~min}$, followed by 30 cycles of $95{ }^{\circ} \mathrm{C}$ for $20 \mathrm{~s}$, $63{ }^{\circ} \mathrm{C}$ for $30 \mathrm{~s}, 72{ }^{\circ} \mathrm{C}$ for $45 \mathrm{~s}$, and a final extension cycle at $72{ }^{\circ} \mathrm{C}$ for $5 \mathrm{~min}$. PCR products were detected on 1,3\% agarose gel in TBE buffer, then were purified with ISOLATE II PCR and Gel Kit (Bioline) and sequenced in two ends (Eurogen, Moscow) using forward and reverse primers. The DNA sequences were aligned with Unipro UGENE software [13]. GenBank sequence accessions are: 'Barke' KX611232, 'Triumph' KX611233, 'Morex' KX611234, 'Franklin' KX789375.

To reveal the $7 \mathrm{bp}$ deletion in exon 1 of HvGA20ox 2 gene $3 \mu \mathrm{l}$ of the PCR product were digested with $1 \mathrm{U}$ of Hinf I (Sibenzyme, Novosibirsk) in a total reaction volume of $15 \mu \mathrm{l}$ containing $1 \mathrm{x}$ SEbuffer $\mathrm{O}(\mathrm{pH}$ 7.6) for $2 \mathrm{~h}$ at $37{ }^{\circ} \mathrm{C}$, followed by electrophoretic separation in $1.5 \%$ agarose gel in TBE buffer.

\section{Results}

Re-sequencing and mapping of HvGA200x2 gene in Morex/Barke DHLs population

To prove that Barke is the $s d w 1 . d$ mutant we resequenced the exon 1 of $H v G A 20 o x 2$ gene for the barley variety. To design primers we aligned the morex_contig_40861 (http://webblast.ipk-gatersleben.de/barley/viroblast.php) containing the published fragment of HvGA200x2 sequence and MLOC_56462.1 that is the coding sequence for $H v G A 20 o x 2$ gene (Fig. 1a). The PCR fragment of 488 bp was successfully amplified and sequenced for Barke, Triumph and Franklin barley varieties which are derived from the barley 'Diamant family' carrying semi-dwarf $s d w 1 /$ denso allele, and also for Morex (tall barley). Deletion of $7 \mathrm{bp}$ leading to the shift of reading frame in Barke, Triumph and Franklin was confirmed in exon 1 of HvGA20ox2. The intact and altered predicted proteins have just 34 amino acid sequence in common, followed by completely different polypeptides (Fig. 1b).

To map the HvGA20ox2 gene we developed the gene specific CAPS marker. The CAPS marker exposes whether a DH line has the 7-bp deletion (Barke allele) or carries intact exon 1 (Morex allele) (Fig. 2). Next, 94 DHLs from the cross of Morex and Barke were assessed with the gene-specific CAPS marker. As the result, the $H \nu G A 200 \times 2$ gene was mapped in the Morex/Barke DHLs population on the long arm of $3 \mathrm{H}$ chromosome in the position of $132.7 \mathrm{cM}$, co-segregating with SNP 1_0754 (Additional file 1).

\section{7-bp deletion in exon 1 of HvGA20ox2 associates with plant height and heading date segregation in Morex/Barke DHLs population}

Segregation of plant height in Morex/Barke DHLs population

To verify whether the $s d w 1 . d$ mutation is associated with the plant height segregation observed in the Morex/ Barke DHLs population, the height of 920 plants (ten individual plants per each of 90 DHLs and two parents (Additional file 2) were compared considering the 7-bp deletion in exon 1 of the HvGA20ox 2 gene. The resulting bimodal distribution is shown in Fig. 3a. Two distinct peaks reflect significant difference between plant height values of the two allele classes $(p<0.0000001)$. For DH lines with Barke $s d w 1 . d$ allele the averaged plant height varied from 50 to $87 \mathrm{~cm}$ with a mean value of $70 \mathrm{~cm}$; for DH lines with Morex allele plant height ranged from 60 to 107, an average $83 \mathrm{~cm}$. It was previously reported that the $s d w 1$ and denso allele reduced height by 10 to $20 \mathrm{~cm}[5,14,15]$.

The plant height variation was also subjected to QTL mapping. As the result, the highly significant QTL peak 


\section{a}

C 40861 CTCCCTCCCTCCCCGATTACAAATACCCCACCATCCCGGACAGCTCTCCCGCACACTCACTCGCAACTAGCTAGCTCACACACCTCTCAT Morex

Barke

Triumph

Franklin CTCCCTCCCTCCCCGATTACAAATACCCCACCATCCCGGACAGCTCTCCCGCACACTCACTCGCAACTAGCTAGCTCACACACCTCTCAT CTCCCTCCCTCCCCGATTACAAATACCCCACCATCCCGGACAGCTCTCCCGCACACTCACTCGCAACTAGCTAGCTCACACACCTCTCAT CTCCCTCCCTCCCCGATTACAAATACCCCACCATCCCGGACAGCTCTCCCGCACACTCACTCGCAACTAGCTAGCTCACACACCTCTCAT

C_ 40861

Morex

Barke

Triumph

Franklin CTCCCTCCCTCCCCGATTACAAATACCCCACCATCCCGGACAGCTCTCCCGCACACTCACTCGCAACTAGCTAGCTCACACACCTCTCAT

C 40861

Morex

Barke

Triumph

CTCATGGTGCTCCAGACCGCTCAGCAAGAACCATCCCTGACGCGCCCGCCTCGCTGCAGCGTCGCCAGCGCGGGCTCGCCGGCCGCCATG СTCATGGTGCTCCACACCGCTCAGCAAGAACCATCCCTGACGCGCCCGCCTCGCTGCAGCGTCGCCAGCGCGGGCTCGCCGGCCGCCATG CTCATGGTGCTCCAGACCGCTCAGCAAGAACCATCCCTGACGCGCCCGCCTCGCTGCAGCGTCGCCAGCGCGGGCTCGCCGGCCGCCATG CTCATGGTGCTCCAGACCGCTCAGCAAGAACCATCCCTGACGCGCCCGCCTCGCTGCAGCGTCGCCAGCGCGGGCTCGCCGGCCGCCATG СTCATGGTGCTCCAGACCGCTCAGCAAGAACCATCCCTGACGCGCCCGCCTCGCTGCAGCGTCGCCAGCGCGGGCTCGCCGGCCGCCATG

Franklin

GACACCAGCCCTGCGACTCCCCTGCTCCTCCAGCCCCCCGCTCCCAGCATTGACCCCTCCGCCGCCAAGGCCGCCGTCAGCAAGGGCGGC GACACCAGCCCTGCGACTCCCCTGCTCCTCCAGCCCCCCGCTCCCAGCATTGACCCCTCCGCCGCCAAGGCCGCCGTCAGCAAGGGCGGC GACACCAGCCCTG------CCTGCTCCTCCAGCCCCCCGCTCCCAGCATTGACCCCTCCGCCGCCAAGGCCGCCGTCAGCAAGGGCGGC GACACCAGCCCTG------CCTGCTCCTCCAGCCCCCCGCTCCCAGCATTGACCCCTCCGCCGCCAAGGCCGCCGTCAGCAAGGGCGGC

C 40861 GGCGCTGCCACCGCGGTGTACGACCTCCGGAGGGAGCCCAAGATCCCCGCCCCGTTCGTGTGGCCGCACGCCGAGGTGCGCCCCACCACG Morex Barke

Triumph GGCGCTGCCACCGCGGTGTACGACCTCCGGAGGGAGCCCAAGATCCCCGCCCCGTTCGTGTGGCCGCACGCCGAGGTGCGCCCCACCACG GGCGCTGCCACCGCGGTGTACGACCTCCGGAGGGAGCCCAAGATCCCCGCCCCGTTCGTGTGGCCGCACGCCGAGGTGCGCCCCACCACG

Franklin GGCGCTGCCACCGCGGTGTACGACCTCCGGAGGGAGCCCAAGATCCCCGCCCCGTTCGTGTGGCCGCACGCCGAGGTGCGCCCCACCACG

C 40861 GCGCAGGAGCTGGCCGTGCCGGTGGTGGACGTGGGCGTGCTGCGCAATGGCGACGCCGCCGGGATACGCCGCGCCGTGGCGCAGGTGGCG

Morex

Barke

Triumph GCGCAGGAGCTGGCCGTGCCGGTGGTGGACGTGGGCGTGCTGCGCAATGGCGACGCCGCCGGGATACGCCGCGCCGTGGCGCAGGTGGCG GCGCAGGAGCTGGCCGTGCCGGTGGTGGACGTGGGCGTGCTGCGCAATGGCGACGCCGCCGGGATACGCCGCGCCGTGGCGCAGGTGGCG

Franklin GCGCAGGAGCTGGCCGTGCCGGTGGTGGACGTGGGCGTGCTGCGCAATGGCGACGCCGCCGGGATACGCCGCGCCGTGGCGCAGGTGGCG

C_40861 TCGGCGTGCGCGACGCACEGGTTCTTCCAGGTGTCCGG

Morex TCGGCGTGCGCGACGCACGGGTTCTTCCAGGTGTCCGG

Barke TCGGCGTGCGCGACGCACGGGTTCTTCCAGGTGTCCGG

Triumph TCGGCGTGCGCGACGCACGGGTTCTTCCAGGTGTCCGG

Franklin TCGGCGTGCGCGACGCACGGGTTCTTCCAGGTGTCCGG

\section{b}

"Morex"-type protein

"Barke"-type protein

"Morex"-type protein

"Barke"-type protein

"Morex"-type protein

"Barke"-type protein

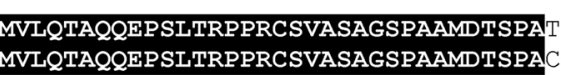

PLLLQPPAPS IDPSAAKAAVSKGGGAATAVYDLRREPKIPAPF SSSPPLPALTPPPPRPPSARAAALPPRCTTSGGSPRSPPRSCG

VWPHAEVRPTTAQELAVPVVDVGVLRNGDAAGIRRAVAQVASACATHGFFQVSGHGVDNALARAALDGASGFFRLPLA RTPRCAPPRRRSWPCRWWTWACCAMATPPGYAAPWRRWRRRARRTGSSRCPGTAWTTPWRARRWTARAGSSVCRWPRS

EKQRARRIPGTVSGYTSAHADRFASKLPWKETLSFGFHDRAGAAAPVVVDYFTSTLGPDYEP

SARGASRGPCPGTRARTPTGSPPSSPGRRPSPSASTTAPAPPRPWWWTTSPAPSGRTTSQ

Fig. 1 7-bp deletion detected in exon 1 of HvGA20ox2 in Barke (KX611232), Triumph (KX611233) and Franklin (KX789375) semi dwarf barley. Medium tall barley Morex (KX611234) has the intact exon 1. Numbers in parenthesis are GenBank accession numbers. a - multiple alignments include morex_contig_40861 (abbreviated as C_40861) that contains the coding sequence of HvGA20ox2 gene (MLOC_56462.1). The start codon is highlighted in gray. Primers positions are indicated by boxes. $\mathbf{b}$ - the predicted protein sequence resulting from 7 bp deletion in exon 1 of HvGA20ox2. The black box indicates 34 amino acids that are in common between intact and altered protein sequence

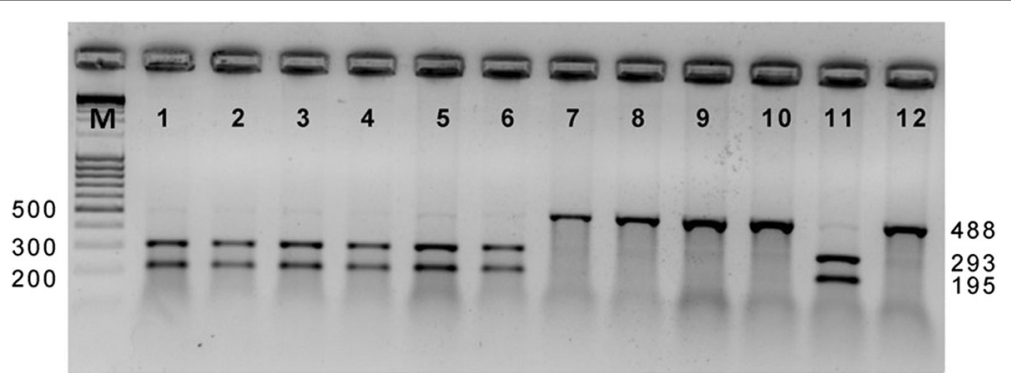

Fig. 2 Genotyping of DHLs derived from Morex and Barke cross with CAPS marker to detect DHLs with the 7-bp deletion in exon 1 of HvGA20ox2 gene (sdw1/denso allele, undigested fragment of $488 \mathrm{bp}$ ). DHLs with the intact exon 1 (Morex allele): lines 1-6, 11. DHLs with sdw1/denso allele: lines 7-10, 12. M - DNA size marker 

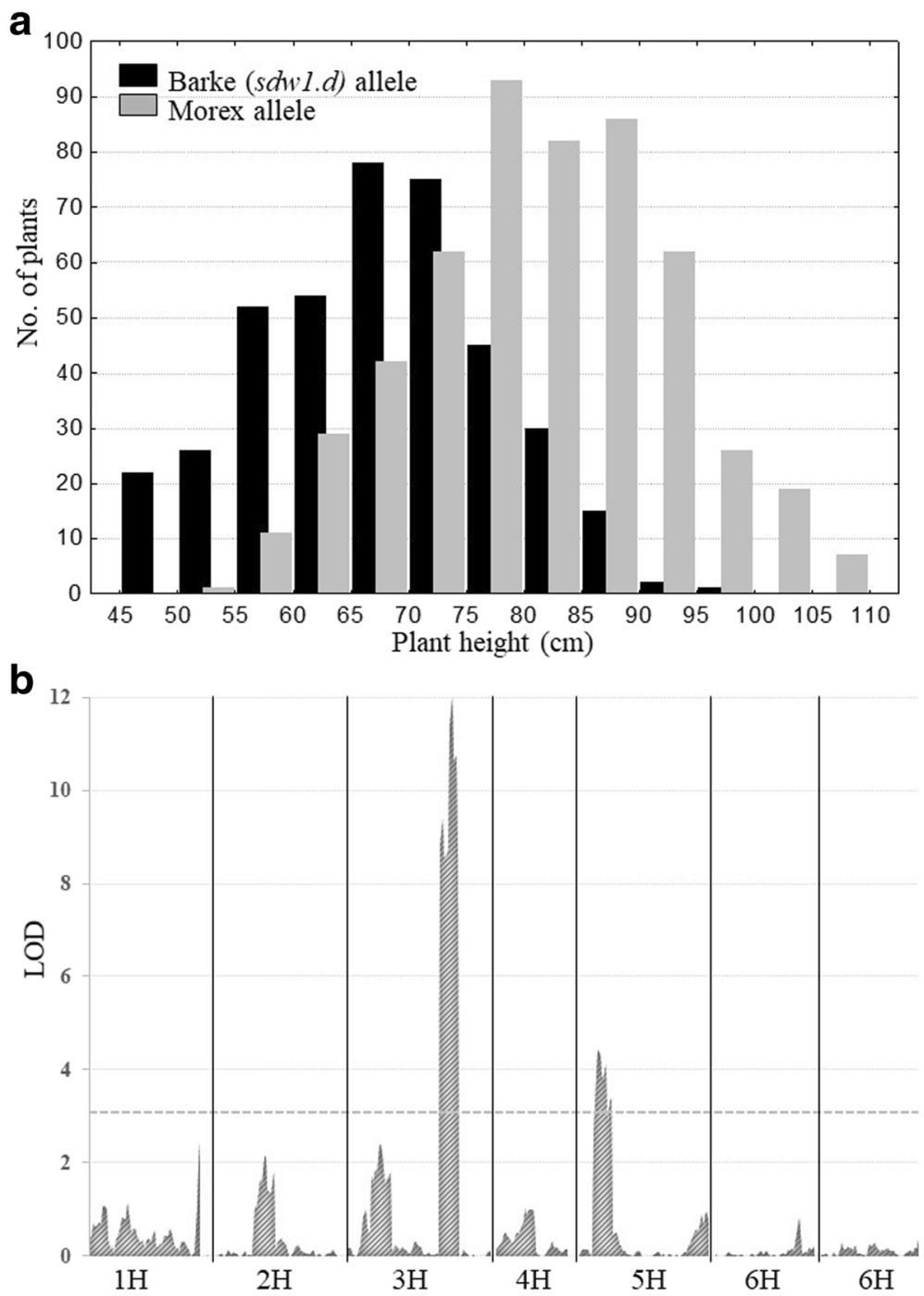

Fig. 3 Influence of sdw1.d allele on plant height segregation in Morex/Barke barley mapping populations. a - bimodal distribution of plant height observed among the progeny of Morex/Barke cross; black bars correspond to the DHLs with 7-bp deletion in exon 1 of HVGA20ox2. b - LOD scans of Composite Interval Mapping (CIM) for plant height in Morex/Barke population in Pushkin (59 59'39" N) in 2015. Barley chromosomes are designated $1 \mathrm{H}$ through $7 \mathrm{H}$. HVGA200x2 gene-specific CAPS is the closest marker to the QTL on $3 \mathrm{H}$

(LOD $=10.6, p<0.05)$ was mapped on $3 \mathrm{H}$ in Morex/ Barke DHLs population, co-segregating with the HvGA20ox2 gene specific CAPS marker and explaining $31 \%$ of observed phenotypical variation (Fig. 3b).

\section{Segregation of heading date in Morex/Barke DHLs population}

The Morex/Barke DHLs population was evaluated for heading date segregation in five environments: three years (2011-2013) in Pushkin and two years $(2012,2013)$ in Krasnodar. The two geographical locations are separated from one another by $15^{\circ}$ of latitude; the day length on the date of seedlings emergence in 2012 was 17 h 30 min and
$13 \mathrm{~h} 24 \mathrm{~min}$ respectively. The averaged heading date of the DH lines in the short day conditions (Krasnodar) was 12-20 days longer than in long day conditions (Pushkin) depending on the year (Additional file 3). The remarkable difference in flowering time was hardly due to alleles of $P p d$ genes, since for both Morex and Barke a recessive allele of $P p d-H 1$ gene was reported, suggesting reduced response to the long day [16].

Two significant QTLs affecting heading date in Morex/Barke DH population were mapped on $2 \mathrm{H}$ and $3 \mathrm{H}$ chromosomes (Fig.4) in two different geographical locations. For both mapped QTLs the prolonged heading date was associated with Barke alleles. One of the QTLs was consistently mapped on the long arm of $3 \mathrm{H}$ in the 

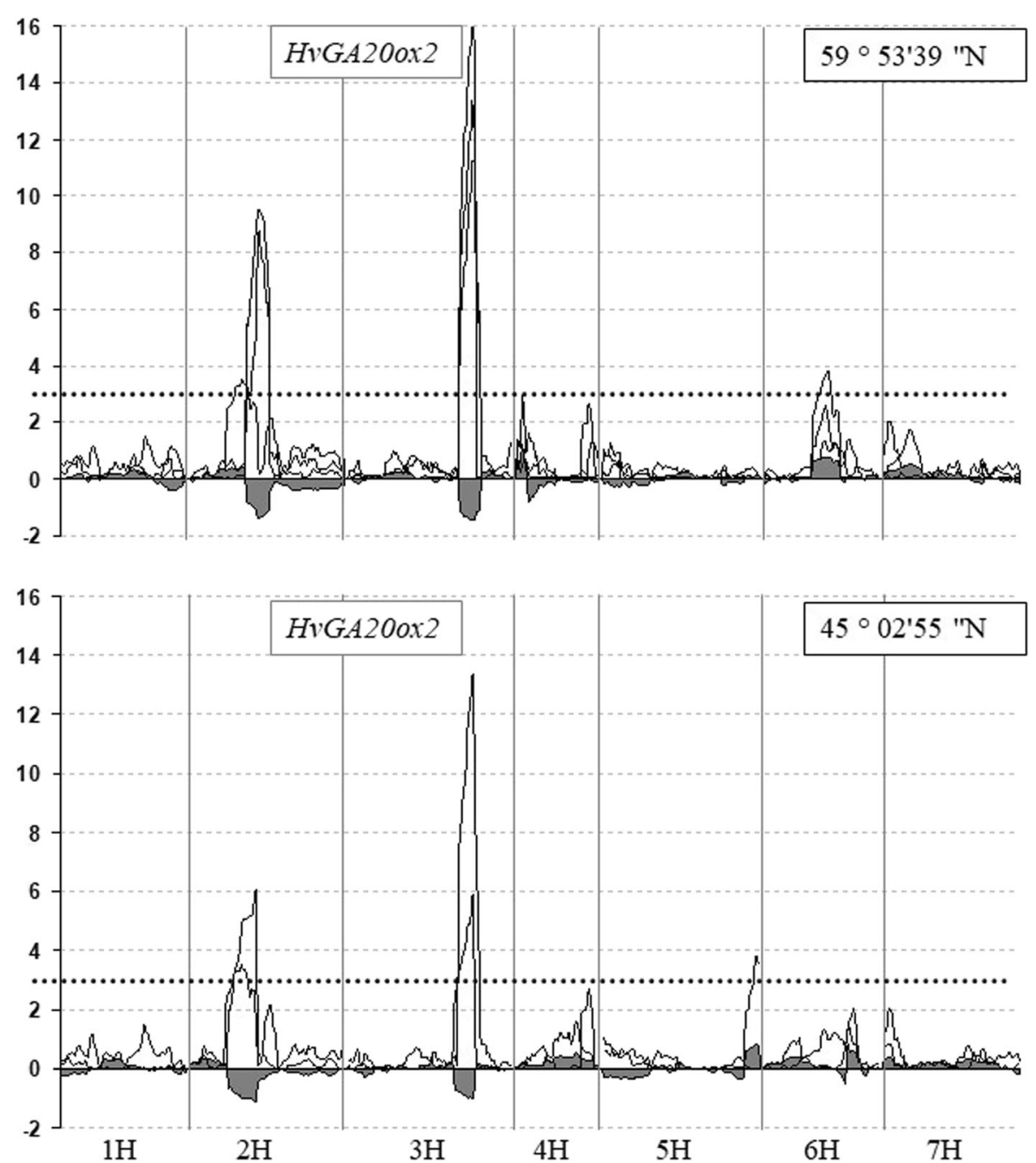

Fig. 4 LOD scans of Composite Interval Mapping (CIM) for days to heading in $90 \mathrm{DH}$ progeny lines derived from the Barke and Morex cross in

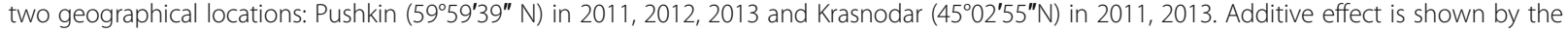
gray fill path along the $\mathrm{X}$ axis. Barley chromosomes are designated $1 \mathrm{H}$ through $7 \mathrm{H}$. HvGA20ox2 gene-specific CAPS is the closest marker to the QTL on $3 \mathrm{H}$ in all environments tested

position of $132.7 \mathrm{cM}$ in all environments tested. The position of the QTL perfectly co-segregated with the HvGA20ox2 gene-specific CAPS marker on Morex/ Barke genetic map. The maximal proportion of variance $\left(R^{2}\right)$ explained by the QTL varied from $28 \%$ to $41 \%$ in two geographical locations. DHLs, inheriting sdw1/denso allele from Barke, initiated flowering 3-5 days later than DHLs carrying the HvGA20ox2 allele from Morex.

The closest SNP marker to the QTL on $3 \mathrm{H}$ in all five environments was SNP 1_0754; this SNP was cosegregating with BOPA SNP marker 11_0977 in OWB barley genetic map [11]. The SNP 11_0977, in turn, was mapped in the same position as a major QTL peak for heading date and plant height detected on $3 \mathrm{H}(139 \mathrm{cM})$ in SBCC145 $\mathrm{x}$ Beatrix $\mathrm{DH}$ population and was proposed to be the closest marker to the $s d w 1 / d e n s o$ dwarfing gene [17].

\section{Variation of thousand grain weight (TGW) and grain quality traits in Morex/Barke DHLs population depending on 7-bp deletion in exon 1 of HvGA200x2 gene}

TGW variation was estimated in the Morex/Barke DHLs population in three environments: Pushkin_2011, Pushkin_2013, Krasnodar_2011. The robust significant QTL peak for the trait was consistently mapped on $2 \mathrm{H}$ chromosome (77.6 cM) (Fig. 5), co-segregating with SNP 3_0896, which was reported as POPA SNP marker of the VRS1 locus controlling the inflorescence type [18]. The allelic state of at the VRS1 locus differentiates the 

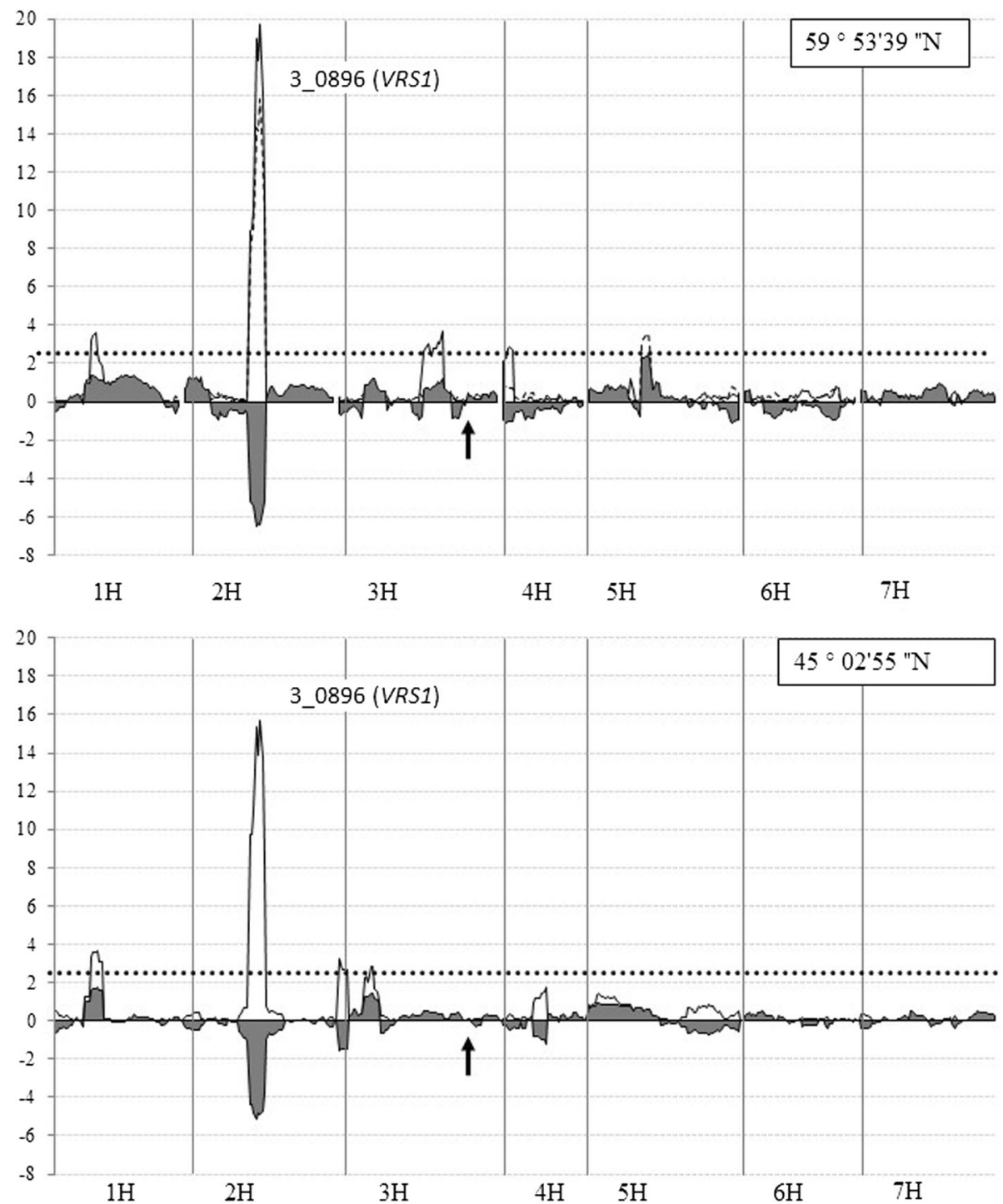

Fig. 5 LOD scans of Composite Interval Mapping (CIM) of thousand grain weight in Morex/Barke barley population in three geographical locations: Pushkin $\left(59^{\circ} 59^{\prime} 39^{\prime \prime} \mathrm{N}\right)$ in 2011,2013 , and Krasnodar $\left(45^{\circ} 02^{\prime} 55^{\prime \prime} \mathrm{N}\right)$ in 2011 . Additive effect is shown by the gray fill path along the X axis. SNP 3_0896 (VRS1) is the closest marker to the QTL on 2H in all environments tested. The position of the sdw1/denso gene is marked by the black arrows

wild type two-rowed barley and six-rowed barley. Lossof-function mutations in the $H v H O X 1$ gene that underlies the VRS1 locus cause a cessation of suppression of lateral-spikelet development and thus lead to the recessive six-rowed phenotype [19]. In the Morex/Barke cross the two-rowed parent Barke carries the dominant Vrs1.b3 allele, while six-rowed parent Morex has the recessive vrs1.a1 allele [18]. Polymorphism in the VRS1 locus explained up to $48 \%$ of the TGW variation observed among the DHLs derived from the cross between Morex and Barke (Additional file 4). The two-rowed DHL offspring showed higher TGW values compared to six-rowed DHLs. However, no impact of the $s d w 1 /$ denso locus on the trait variation was detected.
Grain protein concentration (GPC) is a primary determinant of grain value and malting quality in barley. While very low $\left(<90 \mathrm{~g} \mathrm{~kg}^{-1}\right)$ grain protein concentration reduces the value of malting barley, high grain protein concentration $\left(>145 \mathrm{~g} \mathrm{~kg}^{-1}\right.$ ) is associated with low levels of malt extract and increased likelihood of chill haze in finished beer [20]. Since grain protein concentration is impacted both by environmental conditions and genetic background, it was suggested as an ideal subject for QTL analysis and potential marker assisted selection. Starch content affects water uptake during steeping of barley and is considered as the important trait for malting industry [21]. To check whether $s d w 1 . d$ allele affects GPC and starch content the two traits were assessed in 
Morex/Barke DHLs populations in five and three environments respectively. Alike to TGW analysis results, the 7-bp deletion in exon 1 of $H \nu G A 200 \times 2$ gene did not affected accumulation of protein and starch in grains, while significant influence of VRS1 locus on GPC in Morex/Barke DHLs population was discovered in three out of five environments tested (Additional file 4).

\section{Discussion}

In the present study we investigated effect of $s d w 1 /$ denso allele on the variation of yield-related and malting quality traits in the population of DHLs derived from cross of medium tall barley Morex and semi-dwarf barley Barke. The latter belongs to the 'Diamant family' of the barley varieties inheriting the $s d w 1 /$ denso allele from Xray mutant $\mathrm{cv}$. Diamant. The $s d w 1 /$ denso allele has been reported for more than 150 new successful malting barley cultivars in Europe [22] and also has gained great acceptance in malting barley breeding programs around the world. The question was whether only the short stature of the $s d w 1 /$ denso mutants and their resistance to lodging was the cause for the apparent agronomical success of semi-dwarfing barleys, or the pleiotropic effect of $s d w 1 / d e n s o$ allele on the yield-related traits has played the decisive role. For wheat it was previously reported that the direct effect of the Rht mutations is reduced plant height, but there is also an important pleiotropic effect causing increased assimilate partitioning to developing ears and an increased number of grains per spike and resulting in enhanced yield (reviewed in [4]). On other hand, the agronomic success of $s d-1$ mutants in rice may be intimately linked to the developmental timing of stem growth and panicle development [3].

The $s d w 1 /$ denso locus was mapped on $3 \mathrm{H}$ chromosome of barley few decades ago [23], since that time many QTL mapping studies reported the linkage of the sdw1/denso locus to agronomical important traits (reviewed in [9]). However, the candidate HvGA20ox2 gene for $s d w 1 / d e n s o$ locus and the functional polymorphism assigned to the semi-dwarf alleles were defined just recently $[5,8]$. The $s d w 1 /$ denso allele is most likely resulted from the deletion of $7 \mathrm{bp}$ in exon 1 of $H \nu$ GA20ox2 gene [8]. The deletion leads to the shift of reading frame of $H v G A 200 \times 2$ gene and may affect dramatically the structure of corresponding protein. $H v G A 200 x 2$ encodes GA 20-oxidase that is involved in the final steps of gibberellin biosynthesis. Mutations of the HvGA20ox2 lead to reduced endogenous GAs concentration affecting stem elongation and also flowering time, since GA acts a particularly important developmental switch between vegetative and reproductive development [24]. Corresponding to these reports, we detected the significant association between the 7-bp deletion in exon 1 of $H \nu G A 20 o x 2$ gene and the segregation of plant height and heading date in Morex/Barke DHLs population: DHLs inheriting the $s d w 1 /$ denso allele from Barke were on average $13 \mathrm{~cm}$ shorter in height and initiated flowering 3-5 days later than DHLs carrying the intact $H v G A 200 x 2$ allele from Morex. This also confirms previous observations that the $s d w 1$ barley mutants show a $10-20 \mathrm{~cm}$ reduction of plant height and 3 days heading delay [15, 25]. Besides, Maurer et al. [26] recently highlighted the important role of $s d w 1 /$ denso gene in a whole plant's life cycle: introgression of the wild $s d w 1$ allele into Barke genetic background from wild barley (Hordeum vulgare ssp. spontaneum) increased plant height by up to $12.3 \mathrm{~cm}$, reduced the time required to reach shooting, flowering and maturity by 5.7, 4.3 and 4.0 days, respectively.

While the main characteristics of the $s d w 1 /$ denso phenotype, such as a reduction of culm internode length, increased lodging resistance and delayed flowering are generally recognized, its effect on yield components or malting quality traits in barley is still questionable. QTLs for heading date, growth habit, yield, development score, plumpness and hectolitre weight were co-located with the $s d w 1 /$ denso on the long arm of chromosome $3 \mathrm{H}$ (e.g. $[6,25])$. Both positive and negative effects of $s d w 1 /$ denso gene on the yield components in barley were reported [9, 22]. For example, DHLs derived from the cross of the barley variety Magnum and the variety Goldmarker (carrying the sdw1/denso gene) showed lower plant grain weights and 50 grain weights [27]. Grain yield of semi-dwarf lines derived from the crosses of semi-dwarf 'Royal' parent and eight tall barley lines was not significantly increased in all the cases [15]. The major issue reported for $s d w 1 /$ denso barley varieties is the decreased thousand grain weight: replacement of $s d w 1 /$ denso Barke alleles by those introgressed from wild barley increased TGW by up to $4.5 \mathrm{~g}$ [26]. On other hand, the $s d w 1 /$ denso locus had a large positive effect on grain yield in DHLs population derived from the cross of medium tall AC Metcalfe and semi dwarf Baudin, explaining $49 \%$ of the variation [6].

Final grain yield in wheat and barley depends on grain number and grain weight (reviewed in [4]). The yield components influencing grain number include number of tillers bearing fertile spikes, extension of vegetative as well as reproductive growth, inflorescence architecture, culm hardiness, spike initiation, elongation and branching as well as spikelet formation. In the Morex/Barke segregating population the variation of grain weight (TGW) and grain quality (GPC) were toughly determined by the VRS1 locus, differentiating the two-rowed and six-rowed DHLs progenies. No impact of the $s d w 1 /$ denso locus on the yield component and grain quality traits was detected. 
The ortholog of $H v G A 200 \times 2$ gene in rice genome, $s d 1$ is recognized as the classic Green Revolution gene. Similar to barley, large deletions in coding part of the corresponding rice gene Os20ox-2 were discovered for rice semi-dwarf cultivars. Some of them (i.e. IR8) brought the Green Revolution to many countries in Asia [22] and still show the best agronomic performance [28]. In barley, however, no significant positive pleiotropic effect of $s d w 1 /$ denso gene on the yield and quality components such as TWG or GPC was detected neither in the present study, nor in the most of previous reports. Though, an indirect positive effect of semi dwarfism on agronomic performance in barley could be assumed. Semi-dwarf barleys are more resistant to lodging than tall plants; lodging reduces not only barley yield and grain quality but also affects malt quality since the grain from lodged plants is often lighter in weight and lower in malt extract [29]. Compared to its ancestor cv. Valticky, the $s d w 1 . d$ mutant cv. Diamant was reported to increase grain yields by $12 \%$ [30]. Triumph derived from Diamant is known as the best genetic sources for malting barley in Europe.

The positive or negative effect of $s d w 1 /$ denso gene on the yield components in barley could also depend on different environments. Two-rowed $s d w 1 /$ denso spring barleys could be highly advantageous in Western Europe with the long growing season allowing to accumulate additional biomass that supports higher yields. In hot and dry areas, however, the delay in heading date raises a risk that grains filling will occur concurrently with a drought and high summer temperatures which may harm yield performance of denso-carrying spring barley varieties. As a consequence, lateness in heading associated with denso was reported as another issue of barley breeding in Spain [17].

\section{Conclusions}

In our study the 7-bp deletion in HvGA20ox2 gene, which was recently proposed as the functional polymorphism of $s d w 1 / d e n s o$ locus in barley, was significantly associated with reduced plant height and delayed flowering time in the segregating population of DHLs derived from cross of medium tall barley Morex and semi-dwarf $s d w 1 . d$ ( $s d w 1 /$ denso) variety Barke, independently on environmental cue. On other hand, the sdw1.d mutation did not affect either thousand grain weight or grain quality traits variation in this DHLs population. Thus, the beneficial effect of the semi dwarf $s d w 1 . d$ allele in barley seems does not relate directly to the grain yield potential, but is associated with lodging resistance. Besides, in certain ecological environments the extended period of vegetative growth allows to accumulate additional biomass supporting higher grain yield.

\section{Additional files}

Additional file 1: Mapping of the HVGA200x2 gene in the Morex/Barke DHLs population on the long arm of $3 \mathrm{H}$ chromosome and phenotypic trait data scored for DHLs of the Morex/Barke barley mapping population. (XLSX 27 kb)

Additional file 2: Plant height segregation evaluated for 10 individual plants per each of $90 \mathrm{DHLs}$ and two parents the Morex/Barke DHLs population. (XLSX $15 \mathrm{~kb}$ )

Additional file 3: Flowering time (days to awn emergence) of $90 \mathrm{DHLS}$ derived from Barke and Morex cross in 2011-2013 in two geographical

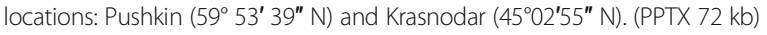

Additional file 4: LOD scans, additive effect and percent of explained phenotypic variation (R2) of Composite Interval Mapping (CIM) performed for agronomic traits scored for DHLs of Morex/Barke barley mapping population. Obtained from output of Windows QTL Cartographer version 2.5 via Perl script. (XLSX $284 \mathrm{~kb}$ )

\section{Acknowledgments}

We are grateful to Prof. Andreas Graner and Prof. Nils Stein for providing the seed material of Morex/Barke DHLs population.

\section{Funding}

This work was supported by Interstate Targeted Program of Eurasian Economic Community "Innovative Biotechnologies" (grant no. 2014-14-M.04-0008). ML was supported by scientific project no. 37.1521.2014/K of the Ministry of Science and Education of the Russian Federation. The publication costs were funded by the Saint Petersburg State University.

Availability of data and materials

Supporting data are included as additional files.

\section{About this supplement}

This article has been published as part of BMC Plant Biology Volume 17 Supplement 1, 2017: Selected articles from PlantGen 2017. The full contents of the supplement are available online at https://bmcplantbiol.biomedcentral.com/ articles/supplements/volume-17-supplement-1.

\section{Authors' contributions}

ST performed the QTL analyses, ML made the sequencing work and designed the CAPS marker, NI OK NV performed phenotyping and the field evaluations, $\mathrm{VH}$ assessed biochemical traits (protein and starch). EP conceived the study and wrote the manuscript. All authors read and approved the final manuscript.

Ethics approval and consent to participate

This study does not contain any research requiring ethical consent or approval.

Consent for publication

Not applicable.

Competing interests

The authors declare that they have no competing interests.

\section{Publisher's Note}

Springer Nature remains neutral with regard to jurisdictional claims in published maps and institutional affiliations.

\section{Author details}

${ }^{1}$ Saint Petersburg State University, Universitetskaya emb.7/9, St. Petersburg 199034, Russia. ${ }^{2}$ N.I. Vavilov Institute of Plant Genetic Resources (VIR), Bolshaya Morskaya, 42-44, 190000 St. Petersburg, Russia. ${ }^{3}$ Saint Petersburg State Forest Technical University, Institutskiy per, 5, 194021 St. Petersburg, Russia. 


\section{Published: 14 November 2017}

\section{References}

1. Borlaug NE. Contributions of conventional plant breeding to food production. Science. 1983;219:689-93.

2. Pearce S, Saville R, Vaughan SP, Chandler PM, Wilhelm EP, Sparks CA, et al. Molecular characterization of Rht-1 dwarfing genes in Hexaploid wheat. Plant Physiol. 2011;157:1820-31.

3. Spielmeyer W, Ellis MH, Chandler PM. Semidwarf (sd-1), "green revolution" rice, contains a defective gibberellin 20-oxidase gene. PNAS. 2002;99(13):9043-8.

4. Nadolska-Orczyk AO, Rajchel IK, Orczyk W, Gasparis S. Major genes determining yield-related traits in wheat and barley. Theor Appl Genet. 2017;130:1081-98.

5. Jia Q, Zhang J, Westcott S, Zhang X, Bellgard M, Lance R, Li C. GA-20 oxidase as a candidate for the semi-dwarf gene sdw1/denso in barley. Funct Integr Genomics. 2009:9:255-62.

6. Jia Q, Zhang XQ, Westcott S, Broughton S, Cakir M, Yang J, Lance R, Li C. Expression level of a gibberellin 20-oxidase gene is associated with multiple agronomic and quality traits in barley. Theor Appl Genet. 2011;122:1451-60.

7. Jia Q, Li C, Shang Y, Zhu J, Hua W, Wang J, Yang J, Zhang G. Molecular characterization and functional analysis of barley semi-dwarf mutant Riso no. 9265. BMC Genomics. 2015;16:927.

8. Xu Y, Jia Q, Zhou G, Zhang XQ, Angessa T, Broughton S, Yan G, Zhang W, L C. Characterization of the sdw1 semi-dwarf gene in barley. BMC Plant Biol. 2017;17(1):11.

9. Kuczyńska A, Surma M, Adamski T, Mikołajczak K, Krystkowiak K, Ogrodowicz P. Effects of the semi-dwarfing sdw1/denso gene in barley. J Appl. Genetics. 2013:54:381-90

10. Standard: GOST 10842. Cereals, pulses and oilseeds. Method for determination of 1000 kernels or seeds weight. http://standards.globalspec.com/std/290913/ gost-10842. (1989). Accessed 22 Dec 1989.

11. Close TJ, Bhat PR, Lonardi S, Wu Y, Rostoks N, et al. Development and implementation of high-throughput SNP genotyping in barley. BMC Genomics. 2009;10:582.

12. Wang S, Basten CJ, and Zeng Z-B. Windows QTL cartographer 2.5 Department of Statistics, North Carolina State University, Raleigh, NC. 2012. http://statgen.ncsu.edu/qtlcart/WQTLCart.htm. Accessed 01 Aug 2012.

13. Okonechnikov K, Golosova O, Fursov M, Unipro UGENE. A unified bioinformatics toolkit. Bioinformatics. 2012;28:1166-7.

14. Grausgruber $H$, Bointner $H$, Tumpold R, Ruckenbauer P, Fischbeck G. Genetic improvement of agronomic and qualitative traits of spring barley. Plant Breed. 2002;121(5):411-6.

15. Hellewell KB, Rasmusson DC, Gallo-Meagher M. Enhancing yield of semidwarf barley. Crop Sci. 2000;40:352-8

16. Turner A, Beales J, Faure S, Dunford RP, Laurie DA. The pseudo-response regulator $\mathrm{Ppd}-\mathrm{H} 1$ provides adaptation to photoperiod in barley. Science. 2005:310:1031-4.

17. Ponce-Molina LJ, Casas AM, Pilar Gracia M, Silvar C, Mansour E, Thomas WBT, Schweizer G, Herz M, Igartua EQTL. Candidate loci for heading date in a large population of a wide barley cross. Crop Sci. 2012;52:2469-80.

18. Cuesta-Marcos A, Szücs P, Close TJ, Filichkin T, Muehlbauer GJ, Smith KP Hayes PM, Genome-wide SNPs and re-sequencing of growth habit and inflorescence genes in barley: implications for association mapping in germplasm arrays varying in size and structure. BMC Genomics. 2010;11(1):707.

19. Komatsuda T, Pourkheirandish M, He C, Azhaguvel P, Kanamori H, Perovic D, Stein N, Graner A, Wicker T, Tagiri A, Lundqvist U, Fujimura T, Matsuoka M, Matsumoto T, Yano M. Six-rowed barley originated from a mutation in a homeodomain-leucine zipper I-class homeobox gene. Proc Natl Acad Sci U S A. 2007;104:1424-9.

20. See D, Kanazin V, Kephart K, Blake T. Mapping genes controlling variation in barley grain protein concentration. Crop Sci. 2009;42(3):680-5.

21. Gupta M, Abu-Ghannam N, Gallaghar E. Barley for brewing: characteristic changes during malting, brewing and applications of its by-products. Compr Rev Food Sci Food Saf. 2010;9(3):318-28.

22. Dockter $\mathrm{C}$, Hansson M. Improving barley culm robustness for secured crop yield in a changing climate. J Exp Bot. 2015;66(12):3499-509.

23. Laurie DA, Pratchett N, Bezant JH, Snape JW. RFLP mapping of five major genes and eight quantitative trait loci controlling flowering time in a winterxspring barley (Hordeum vulgare L.) cross. Genome. 1995;38:575-85.

24. Eriksson S, Bohlenius H, Moritz T, Nilsson O. GA4 is the active gibberellin in the regulation of LEAFY transcription and Arabidopsis floral initiation. Plant Cell. 2006;18:2172-81.
25. Kuczyńska A, Mikołajczak K, Ćwiek H. Pleiotropic effects of the sdw1 locus in barley populations representing different rounds of recombination. Electron J Biotechnol. 2014;17(5):217-23.

26. Maurer A, Draba V, Pillen K. Genomic dissection of plant development and its impact on thousand grain weight in barley through nested association mapping. J Exp Bot. 2016;67(8):2507-18.

27. Laurie DA, Pratchett N, Romero C, Simpson E, Snape JW. Assignment of the denso dwarfing gene to the long arm of chromosome $3(3 \mathrm{H})$ of barley by use of RFLP markers. Plant Breed. 1993:111:198-203.

28. Maluszynski M, Szarejko I. Induced mutations in the green and gene revolutions. In: Tuberosa R, Phillips RL, Gale M. In the wake of the double helix: from the green revolution to the gene revolution. Bologna: Avenue media; 2005. p. 403-425.

29. Day $A D$, Dickson AD. Effect of artificial lodging on grain and malt quality of fall-sown irrigated barley. Agron J. 1958:50(6):338-40.

30. Ahloowalia B, Maluszynski M, Nichterlein KE. Global impact of mutationderived varieties. Euphytica. 2004:35(2):187-204.

\section{Submit your next manuscript to BioMed Central and we will help you at every step:}

- We accept pre-submission inquiries

- Our selector tool helps you to find the most relevant journal

- We provide round the clock customer support

- Convenient online submission

- Thorough peer review

- Inclusion in PubMed and all major indexing services

- Maximum visibility for your research

Submit your manuscript at www.biomedcentral.com/submit
Biomed Central 